\title{
Item learning in cognitive skill training: Effects of item difficulty
}

\author{
WILLIAM J. HOYER, JOHN CERELLA, and SERGE V. ONYPER \\ Syracuse University, Syracuse, New York
}

\begin{abstract}
Item difficulty effects in skill learning were examined by giving participants extensive training with repeated alphabet arithmetic problems that varied in addend size (e.g., $\mathrm{C}-\mathrm{D}=$ ? is easy; $\mathrm{C}-\mathrm{J}=$ ? is harder). Recognition memory for the items, as measured by interpolated recognition tests, was acquired early in training and was unaffected by item difficulty. Memory for the solutions to items, as measured by the participants' strategy reports that they had retrieved, rather than computed, the solution, was acquired later and was affected by item difficulty. Solutions to easier items were learned earlier in training for both young adults (18-24 years) and older adults (60-75 years), superimposed on an overall lower level of solution learning in older participants. The results suggest that the formation of associations between problems and their solutions is effortful and shares limited processing resources with the computational demands of the problem.
\end{abstract}

For skills that initially require the execution of an algorithm (e.g., dot counting or alphabet arithmetic), practice can produce a strategy shift to direct memory retrieval when the stimuli are repeated (e.g., Compton \& Logan, 1991; Delaney, Reder, Staszewski, \& Ritter, 1998; Haider \& Frensch, 2002; Logan, 1988; Rickard, 1997). In alphabet arithmetic, for example, suppose that a person is taught to solve problems that involve calculating the numeric difference between two letters (e.g., $\mathrm{C}-\mathrm{J}=$ ?; the answer is 7 , letting $\mathrm{A}=1, \mathrm{~B}=2$, etc.). If problems are repeated and if the person remembers particular problems and their solutions, the type of processing carried out on repeated trials may shift from calculation to retrieval.

That such shifts actually occur has been inferred from several features of the response latencies, over and above the general speedup in the carrying out of mental operations that is associated with practice, described by Newell and Rosenbloom (1981) and others. Response times (RTs) increase linearly with numerosity or addend size in counting and alphabet enumeration tasks. When stimuli are repeated, the slope of the load function declines over the course of training, often flattening to zero, suggesting a shift from calculation to retrieval. This interpretation has been reinforced by findings that trained items remain load free and novel items show load effects

Part of this research was presented as a poster at the 43rd Annual Meeting of the Psychonomic Society, November 2002, in Kansas City. This research was supported by Research Grant AG11451 from the National Institute on Aging. We thank Aaron Benjamin, Marilyn Hartman, Marc Howard, and Martin Sliwinski for comments on an earlier draft and Korena Onyper and Greg Mangan for assistance with data collection. Correspondence should be addressed to W. J. Hoyer, Department of Psychology, Syracuse University, Syracuse, NY 13244-2340(e-mail: wjhoyer@syr.edu). when transfer is assessed (e.g., Barrouillet \& Fayol, 1998; Logan, 1988; Logan \& Klapp, 1991; Thevenot, Barrouillet, \& Fayol, 2001).

In addition to the block-by-block analysis of RTs, strategy shifts have been documented by probing participants, immediately after a response to a skill problem, as to whether they computed or retrieved (e.g., Compton \& Logan, 1991; Delaney et al., 1998; Logan, 1988; Rickard, 1997). The probability of retrieve reports increases with training. Distributions of RTs can then be sorted by strategy; RTs labeled by the participant as computes are long and load sensitive, and RTs labeled as retrieves are short and load free.

This research leads to the conclusion that one of the primary determinants of performance on a skill trial is whether the current item is computed or retrieved. We call the shift from computation to retrieval item learning. Little is known about the factors that might influence the course of item learning. Typically, learning is averaged across items, on the assumption that the items making up the experimental task are roughly homogeneous in their learnability. Only two studies known to us have introduced systematic differences between items and have tracked the consequences for skill learning. Nosofsky and Palmeri (1997; Palmeri, 1997) manipulated interstimulus similarity and examined its effect on the magnitude of the repetition effect. They found that high similarity facilitated the learning of items that mapped to the same response and that it retarded the learning of items that mapped to different responses, analogous to the effects seen in studies of stimulus categorization.

In the study reported here, a different kind of item factor was taken up, that of computational difficulty. We used an alphabet arithmetic task and manipulated the computational difficulty of items within a problem set by varying the magnitude of the numeric difference between 
the two letters making up a problem, referred to as the addend size. A set of 12 alphabet arithmetic problems was composed whose addend sizes ranged from 2 to 7 . The problem set was presented repeatedly until $75 \%$ of the items were consistently retrieved. Item recognition, as well as item learning, was assessed. The acquisition of item or stimulus information was measured by three item recognition tests presented after the third, sixth, and ninth blocks. Item learning was measured by strategy probes given after every correct response during skill learning. That is, the participants reported whether they had computed the solution for that item or had retrieved the solution from memory.

How might problem difficulty be expected to affect item recognition and the learning of particular items within a repeated set of problems? In Logan's (1988, 1992) and Rickard's (1997) models of cognitive skill acquisition, item learning occurs through the formation or strengthening of memory traces in an automatic fashion. If trace formation were truly automatic and independent of the concurrent demands placed on processing resources, the difficulty of the item should have no effect on item acquisition. But other alternatives are plausible. If trace formation were capacity demanding, then as compared with easier items, harder items would be acquired later in the course of training or would be remembered less well, because of the concurrent processing load at the time of exposure. On this hypothesis, the participant is in a dual-task situation, with computation as the primary task and trace formation as the secondary task. It has been reported in numerous memory studies that the more demanding the primary task, the greater the cost to the secondary task (e.g., Craik, Naveh-Benjamin, Ishaik, \& Anderson, 2000). However, the opposite hypothesis can also be entertained: Harder items may benefit from a form of deep processing or enriched encoding, in which case such items ought to be remembered earlier and/or better (e.g., Craik, 2002).

For our study, older adults were tested, as well as college-aged adults, because of known differences in the learning performance of these age groups. Specifically, there is much research that suggests that older adults have reduced cognitive resources, as compared with young adults. Meta-analyses of age-cognition relations have revealed effects that are best accounted for by agerelated limitations in processing resources that affect performance across many kinds of cognitive tasks (e.g., Cerella, 1990; Verhaeghen \& Salthouse, 1997). An implication of this process-general account is that older adults should show increased costs in dual-task procedures, and such findings have been obtained in several investigations (e.g., N. D. Anderson, Craik, \& Naveh-Benjamin, 1998; Kramer, Larish, \& Strayer, 1995; Naveh-Benjamin, 2000). In our study, if item learning is found to be capacity demanding for younger adults, so that harder items are acquired less well, the effect should be observed in a magnified form in older adults because of reduced processing resources.
It is also well established that older adults show markedly reduced levels of episodic memory, as revealed, say, in list recall procedures. One component of this age loss is hypothesized to reflect an encoding deficit - that is, a poorly elaborated memory trace. Support for this position can be derived from studies showing that older participants benefit more from orienting tasks that require deep or elaborative encoding than do young participants (e.g., N. D. Anderson et al., 1998). Thus, if item learning is found to benefit from harder calculations, this effect should be observed in magnified form in older adults. Thus, if computational demand either helps or hurts trace formation, the effect should be easier to observe, because of magnified effects, in older adults. Analogous age amplification has been reported for treatments that benefit memory trace formation (Castel, Benjamin, Craik, \& Watkins, 2002), as well as for treatments that disrupt trace formation (N. D. Anderson et al., 1998; Naveh-Benjamin, 2000).

A second reason for testing younger and older adults in this study was to contribute to the understanding of age differences in skill learning. Age-related differences in skill learning are well established (e.g., Charness \& Campbell, 1988; Hashtroudi, Chrosniak, \& Schwartz, 1991; Jenkins \& Hoyer, 2000; Rogers, Hertzog, \& Fisk, 2000; Salthouse, 1994; Salthouse \& Somberg, 1982; Siegler \& Lemaire, 1997; Strayer \& Kramer, 1994; Touron, Hoyer, \& Cerella, 2001, 2003). We hope to provide support for the view that age differences in skill learning can be attributed to an age deficit in the retrieval of past solutions.

\section{METHOD}

\section{Participants}

Thirty-one younger adults (18-24 years) and 28 older adults (60-75 years) were tested. Younger adults were recruited from the human participants pool of the Department of Psychology at Syracuse University and received course credit for their participation. Older adults were community-residing volunteers recruited from the registry of the Adult Cognition Laboratory at Syracuse University. Older adults were paid $\$ 30$ for their participation. Prior to testing, the participants reported their education level and their overall physical health, using a 5-point scale. Individuals who reported that they were not taking any medications known to affect memory or learning, who rated their health as average, good, or excellent (ratings of 3,2 or 1, respectively), and who had corrected or noncorrected near visual acuity of 20/30 or better were eligible.

The Digit Span subtest of the Wechsler Adult Intelligence ScaleRevised (Wechsler, 1981), the Symbol Digit Test (Smith, 1982), the Number Comparisons subtest from the Kit of Factor Referenced Cognitive Tests (Ekstrom, French, Harman, \& Dermen, 1976), Turner and Engle's (1989) measure of operational span, and several other brief tests were administered for the purpose of describing the age samples. Means and standard deviations for these measures are reported in Table 1.

\section{Stimuli and Procedures}

The participants were given alphabet arithmetic problems, for which the correct solutions could be obtained by determining the arithmetic difference between two letters of the alphabet (inclusive of the second letter) and keying in the result. To illustrate, the cor- 
Table 1

Means (and Standard Deviations) for Measures of the Characteristics of the Research Participants

\begin{tabular}{lrrrrr}
\hline & \multicolumn{2}{c}{ Younger Adults } & & \multicolumn{2}{c}{ Older Adults } \\
\cline { 2 - 3 } \cline { 5 - 6 } & \multicolumn{1}{c}{$M$} & $S D$ & & $M$ & $S D$ \\
\hline$N$ & 31 & & 28 & \\
Age** $_{\text {Education }}$ & 20.4 & 1.7 & 72.2 & 4.9 \\
Health & 14.3 & 1.5 & 14.8 & 2.1 \\
Digit span & 1.9 & 0.6 & 2.1 & 0.7 \\
Number comparisons** & 16.5 & 3.7 & 16.8 & 3.8 \\
Number ordering & 25.9 & 5.4 & 20.9 & 4.5 \\
Object ordering* & 16.3 & 4.0 & 15.6 & 3.3 \\
Operational span* & 5.3 & 1.0 & 4.6 & 0.8 \\
Symbol digit** & 22.3 & 10.7 & 15.7 & 7.0 \\
\hline
\end{tabular}

Note-Significant group differences are indicated with asterisks: $* p<$ $.01 ; * *<.001$. Education, self-reported number of years of formal education; health, self-reported using a scale from 1 (excellent) to 5 (poor); digit span, measure combines the forward span and backward span scores for the WAIS-R digit span subtest (Wechsler, 1981); number comparisons, score on the Number Comparisons subtest from the Kit of Factor Referenced Cognitive Tests (Ekstrom, French, Harman, \& Dermen, 1976); number ordering, score on a span measure of working memory in which strings of digits are reordered numerically (Onyper $\&$ Hoyer, 2003); object ordering, score on a span measure of working memory in which names of items are reordered in terms of size (from Cherry \& Park, 1993); operational span, score on a span measure of working memory in which words memorized while solutions to simple mathematical equations are verified are recalled (Turner \& Engle, 1989); symbol digit, number of symbol-to-digit substitutions completed in $90 \mathrm{sec}$ (Smith, 1982).

rect response to the problem $\mathrm{D}-\mathrm{G}=$ ? was to press 3 on the numeric keypad. We describe this problem as having an addend size of 3. Problem sets were composed of 14 items, 12 experimental problems, two each of addend sizes 2, 3, 4, 5, 6, and 7, and two end effects problems, one at addend 1 and one at addend 8 . The responses to problems with addends of 1 and 8 were not included in the analysis of results. To control for mnemonic idiosyncrasies and for differences tied to particular letter positions, problems were selected randomly from the set of all possible problems for each participant (excluding problems that began with A).

The problem set was presented repeatedly in 14-trial blocks. Order of presentation was random within blocks, and the participants were given an opportunity to take rest breaks after each block. Instructions emphasized both speed and accuracy. The participants were given practice and demonstrated proficiency in keyboard use and in solving practice problems prior to testing.

A program in Visual Basic 6 was written to control stimulus presentation and the recording of RTs. Each trial began with a 500-msec fixation cross, followed by the presentation of one of the problems. The problems were presented in a horizontal format at the center of the computer screen at eye level and subtended $6^{\circ}$ of visual angle at a viewing distance of $50 \mathrm{~cm}$. The stimulus remained on the screen until a keypress response was made. If the response was incorrect, an error message was presented for $500 \mathrm{msec}$, followed by a 1,000msec blank screen before the next problem appeared. If the response was correct, it was followed by a 500-msec blank screen, and then a strategy probe was presented.

The strategy probe requested an introspective report from the participant as to whether the response just made was determined by a computation, by memory retrieval of the solution, or otherwise. Three keys on the computer keyboard (the A, S, and D keys) were labeled $C, M$, and $O$ for the participant to respond compute, memory, or other to the probe. Previous studies have demonstrated that such probes serve to validly partition responses by solution strate- gies (Rickard, 1997; Touron et al., 2003). A 1,000-msec blank screen followed the strategy response, after which the next problem (or a rest break prompt) was presented.

Three on-screen recognition tests were administered, one each after the third, sixth, and ninth blocks of testing. These tests consisted of 24 items, the 12 items making up the experimental problem set and 12 never-presented problems, displayed on a single screen in a mixed, random order. The never-presented problems were unique in each recognition test. The participants were instructed to use the mouse to make checks next to the items they had seen before and were required to check exactly 12 items. The tests were untimed.

The session was terminated after the participant reached a $75 \%$ item-learning criterion, defined by strategy reports of at least nine retrieves in each of two consecutive blocks. Retrieve responses to the end effect items were not counted toward the learning criterion, and the session was terminated after 80 blocks if the $75 \%$ criterion had not been achieved.

\section{RESULTS}

Our primary interest was in examining the effects of addend size on item learning, measured by the mean proportion of retrievals, as opposed to computes, found in the strategy probes, and the effects of addend size on item recognition, measured by the mean proportions of items recognized on each of the recognition tests. Secondarily, the pattern of RTs as a function of addend size was examined for computes and retrieves, as a check on the validity of the strategy reports and to assess age-related slowing. The data from 1 young participant who took unusually long to reach criterion were excluded from all of the analyses, and the recognition test data from 1 other young participant were not usable.

\section{Blocks to Criterion}

On average, older participants took substantially longer to meet the $75 \%$ learning criterion (53 blocks of training) than did the college-aged participants (19 blocks). There was also a considerable range of finishing points within each age group (young, 11-33 blocks; old, 11-80 blocks). In light of these findings, we decided to examine item-learning effects separately for early learners and late learners within each age group. Accordingly, the 59 participants were divided into four subgroups (young/old $\times$ early/late) for most of the analyses that follow. Rather than performing a median split to partition the sample, we decided to follow naturally occurring clusters in the distribution of finishing points. On the basis of clustering in the blocks-to-criterion measure, we defined early-young learners as those who met the criterion in $11-17$ blocks $(n=12)$ and late-young learners as those who met the criterion in 18-33 blocks ( $n=$ $19)$. Early-old learners were defined as those who met the criterion in 11-28 blocks $(n=9)$, and late-old learners as those who met the criterion in 50-80 blocks ( $n=$ $19)$. Seven participants assigned to the late-old subgroup were terminated at 80 blocks without meeting the criterion; each of the participants who failed to reach criterion reported some retrieves. Within each age group, 
early and late learners did not differ significantly in age, education, health, or any of the measures administered as part of the cognitive battery.

Defined in this way, the effect of subgroups on blocks to criterion was necessarily significant $[F(3,54)=$ $\left.206.70, M S_{\mathrm{e}}=51.726, p=.001\right]$. Scheffé post hoc tests confirmed that the early-young participants learned in fewer blocks $(M=13.58, S D=2.1)$ than did the late-young ones $(M=22.72, S D=1.7 ; p<.01)$. Similarly, early-old participants took fewer blocks $(M=$ $19.89, S D=2.4)$ than did late-old ones $(M=68.89$,
$S D=1.7 ; p<.001)$. Blocks to criterion were not significantly different for the late-young and the early-old groups. The survival curves for the four subgroups are shown in the lower panel of Figure 1 as a function of training blocks.

\section{Item Learning}

The upper panel of Figure 1 shows the mean proportion of retrievals for each subgroup. This proportion was calculated by dividing the number of retrieval reports by the total number of strategy probes for each block for
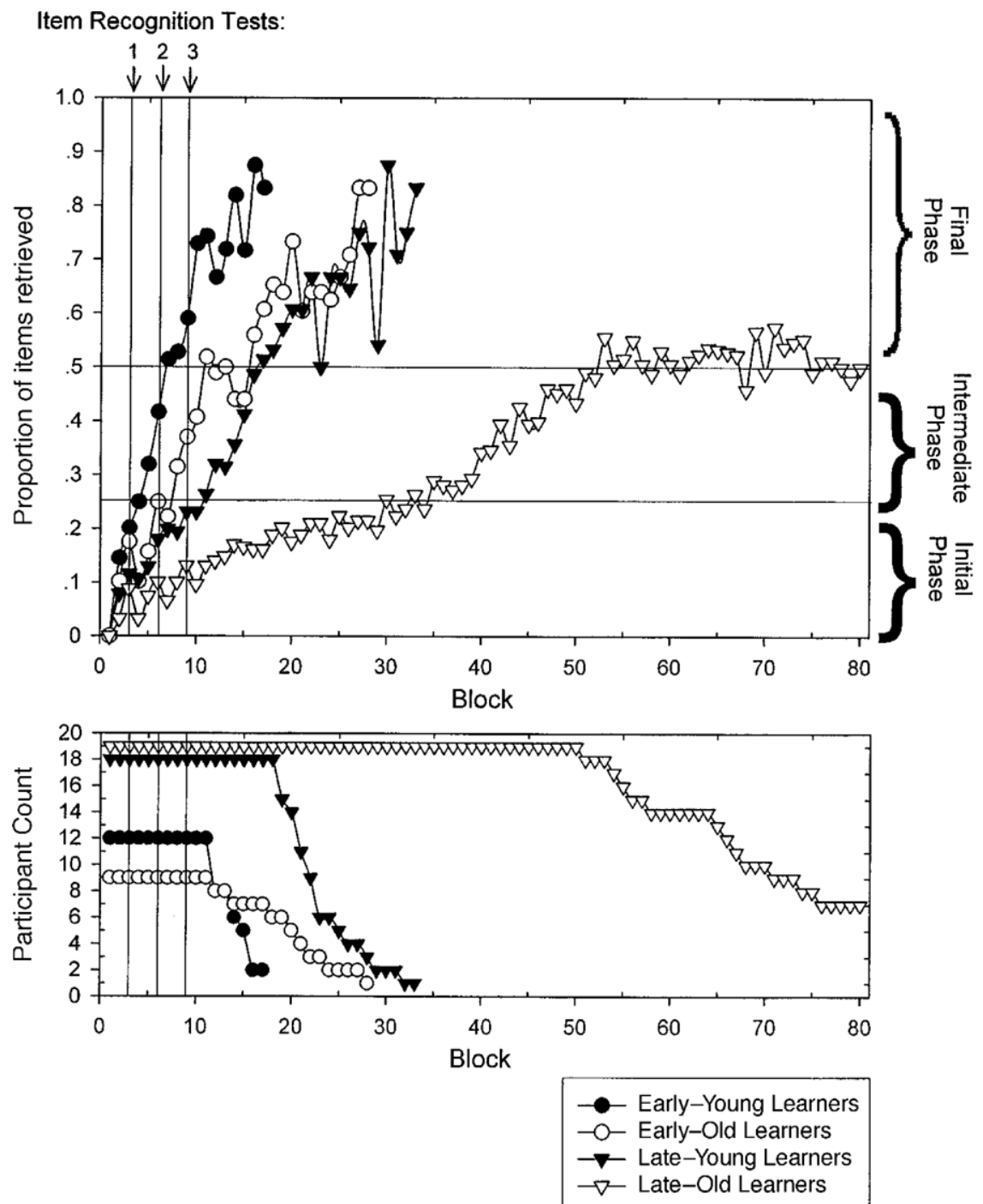

Figure 1. Proportions of items retrieved by groups as a function of blocks. In the upper panel, the horizontal lines indicate the ranges for the initial, intermediate, and final phases of acquisition, and the vertical lines indicate the three administrations of the recognition test. In the lower panel, the participant counts (survival curves) by groups and blocks indicate the differences in attaining the $75 \%$ learning criterion. Seven older adults did not reach the criterion in the 80 blocks allotted for the session. Because their item acquisition data highly resembled the old-late learner data, these 7 participants were included with the late-old learners, who did attain the $75 \%$ criterion. The late-old curve in the lower panel reflects this artifact of data analysis, since it stops at Block 80 and indicates that 7 late-old participants never reached the stopping criterion. 
each participant and averaging across participants. As can be seen in the figure, the item acquisition curve for early-young learners rises rapidly, the curves for late-young and early-old learners largely overlap, and the curve for late-old learners rises slowly.

Superimposed on Figure 1 are two horizontal bands demarcating three phases of learning. The initial phase was defined by all the blocks in which the average retrieval proportions of a participant fell in the range $.01-.25$ (1-3 retrieves out of the 12 possible in a block). The intermediate phase was defined by all the blocks with .26-.50 retrieval proportions (4-6 retrieves). The final phase was defined by all the blocks with .51-1.00 retrieval proportions (7-12 retrieves). Note that few final phase blocks exceeded nine retrieves, which was the termination cutoff. The 7 late-old participants who failed to meet the learning criterion nonetheless contributed substantially to the final phase data: These 7 participants had an average of 9 final phase blocks, as compared with an average of 6 final phase blocks from individuals in the other three learning groups. Also note that there were very few regressions in the number of retrievals reported by the participants. No such regressions occurred for any of the younger adults. For older adults, there was an average of 0.025 drops for early learners and 0.05 for late learners, and these drops occurred only on trials immediately after the recognition tests. By recognition tests, across early/late subgroups, the data for older adults were as follows: For Test 1, there was an average drop of less than one item in Block 4, followed by full recovery in Block 5; for Test 2, there was an average drop of less than half an item in Block 7 and full recovery in Block 8; for Test 3, there was an average drop of less than half an item in Block 10 and full recovery in Block 9.

The effects of addend on the mean proportion of retrievals are shown by phase of learning in the four panels of Figure 2, one panel for each of the four subgroups. In each panel, it appears that smaller addends were learned more readily than larger addends. These effects were attenuated as learning progressed, due to saturation at ceiling. These trends were confirmed in a $4 \times 3 \times 6$ mixed analysis of variance (ANOVA) with subgroup, phase, and addend as factors. The significant main effects of phase $\left[F(2,108)=683.88, M S_{\mathrm{e}}=0.036, p<.001\right]$ and addend $\left[F(5,270)=19.88, M S_{\mathrm{e}}=0.137, p<.001\right]$ were qualified by two significant two-way interactionssubgroup $\times$ phase $[F(6,324)=3.91, p<.001]$ and phase $\times$ addend $\left[F(10,540)=2.80, M S_{\mathrm{e}}=0.035, p<\right.$ $.002]$. Decomposition of the subgroup $\times$ phase interaction indicated that in Phase 3, there was less retrieval for the late-old learners than for the early-young learners. This was due to the fact that 7 of the 28 older participants failed to meet the $75 \%$ learning criterion. The phase $X$ addend interaction reflects the waxing and waning of the addend effect as it emerged from the accuracy floor of Phase 1 and merged with the accuracy ceiling of Phase 3. More noteworthy is the fact that both the subgroup $\times$ addend interaction $[F(15,270)=1.20]$ and the subgroup $\times$ phase $\times$ addend interaction $[F(30,540)<$
1.00] failed to reach significance; thus, the magnitude of the addend effect was unaffected by differences in age or in learning rate. No other interactions in this analysis were significant (all $p \mathrm{~s}>.1$ ).

Although the phase plots for the younger and the older adults did not differ, Figure 1 shows that the older participants required considerably more training to attain each phase. This outcome can be seen plainly in Figure 3 , which combines early and late subgroups so as to expose the age difference alone. The figure shows the proportion of items retrieved over the first 12 blocks. This range allowed us to include all the participants; beyond Block 12, increasing numbers of participants met the learning criterion and terminated (5 participants terminated at Block 11 , so the Block 12 values for these participants were estimated by linear extrapolation from their data for Blocks 10 and 11). Analysis of these data revealed significant main effects of age $[F(1,56)=10.28$, $\left.M S_{\mathrm{e}}=0.026, p<.002\right]$ and blocks $[F(11,616)=37.63$, $\left.M S_{\mathrm{e}}=0.021, p<.001\right]$ and a significant age $\times$ blocks interaction $\left[F(11,616)=4.09, M S_{\mathrm{e}}=0.021, p<.001\right]$.

At first sight, the age $\times$ blocks interaction seems to suggest an age deficit in the rate of item learning. Note, however, that the progress of item learning was disrupted in the older participants by the recognition tests administered between Blocks 3 and 4, 6 and 7, and 9 and 10. Retrieval reports fell immediately after each recognition test and climbed again until the next test was administered. Between tests, the item-learning rate of the older group appears to parallel that of the younger group. Some or all of the age difference in the overall learning rate may be attributable to the disruptive effect of the interim tests.

This interpretation was tested in an ANOVA in which the block factor was replaced by a test cycle factor that partitioned Blocks 1-12 into four levels defined by the recognition tests (Level 1, Blocks 1-3; Level 2, Blocks 4-6; Level 3, Blocks 7-9; Level 4, Blocks 10-12) and by an interstep factor with three levels, corresponding to the $1 \mathrm{st}, 2 \mathrm{nd}$, and $3 \mathrm{rd}$ block within each cycle. In this analysis, the within-subjects main effects of test cycle $\left[F(3,168)=51.67, M S_{\mathrm{e}}=0.05, p<.001\right]$ and interstep $\left[F(2,112)=37.84, M S_{\mathrm{e}}=0.014, p<.001\right]$ and the age $\times$ cycle interaction $\left[F(3,168)=6.06, M S_{\mathrm{e}}=0.05\right.$, $p<.001]$ were significant. Decomposition of the interaction indicated significant improvements between successive test cycles $(p<.01)$ and significant age declines at each cycle $(p<.05)$. The age $\times$ step interaction was not significant $[F(2,112)<1]$, indicating that learning rates did not differ between the younger and the older adults within a cycle. The combination of results suggests that the administration of the recognition tests served to disrupt item retrieval for the older adults, but not for the younger adults.

\section{Item Recognition}

The effect of subgroup on recognition test performance was significant $\left[F(3,53)=8.34, M S_{\mathrm{e}}=0.05, p<\right.$ $.001]$, indicating that the early-young participants did not differ from the late-young ones $(p=1)$ and that the 


\section{Early-Young Learners}

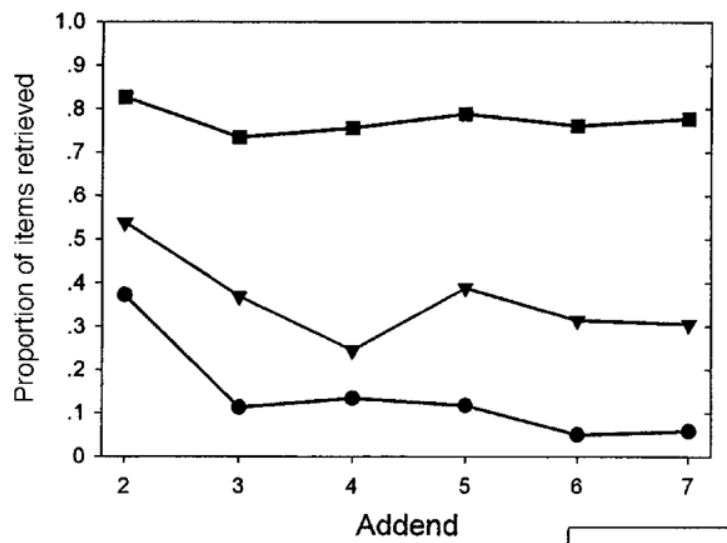

\section{Late-Young Learners}

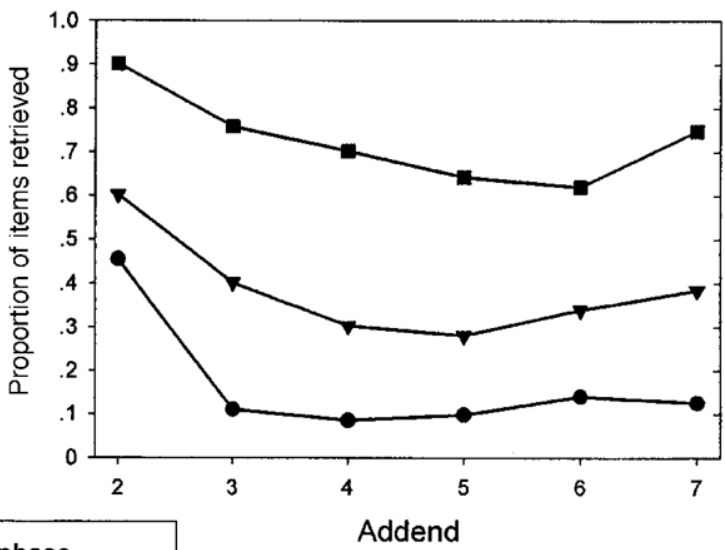

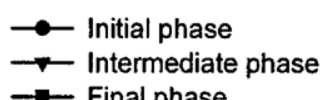

$\rightarrow-$ Final phase

Early-Old Learners

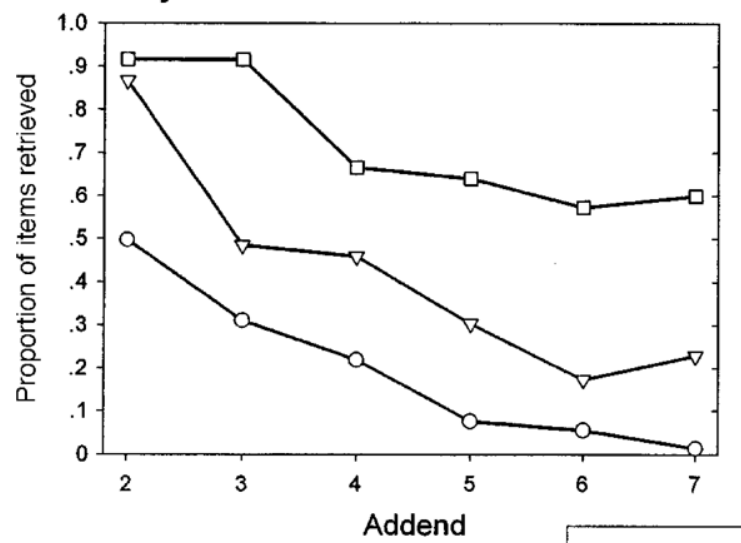

Late-Old Learners

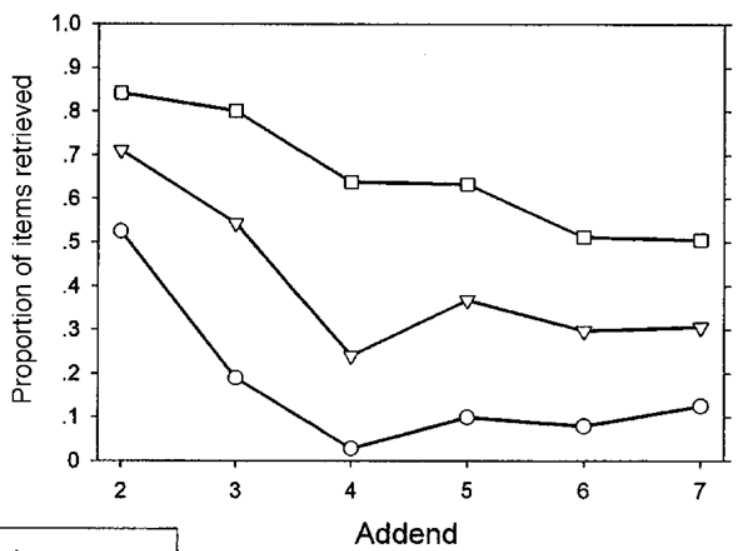

$$
\begin{aligned}
& -\infty \text { Initial phase } \\
& \rightarrow-\text { Intermediate phase } \\
& \rightarrow-\text { Final phase }
\end{aligned}
$$

Figure 2. Proportions of items retrieved by phase of acquisition and addend for each subgroup: early-young, late-young, early-old, and late-old.

early-old participants did not differ from the late-old ones $(p=.220)$ in the overall levels of item recognition. Consequently, item recognition proportions were collapsed across age subgroups and were analyzed using a three-way mixed ANOVA with age (2), addend (6), and test number (3) as factors. The proportions of items accurately recognized were higher for younger adults (.80) than for older adults $\left[.72 ; F(1,56)=16.71, M S_{\mathrm{e}}=\right.$ $0.005, p<.001]$. The main effect of test number was significant $\left[F(2,112)=19.88, M S_{\mathrm{e}}=0.053, p<.001\right]$. Disregarding age, a higher proportion of items was recognized on Test $3(.80)$ than on Test $2(.76 ; p=.031)$ or Test $1(.70 ; p<.001)$. The interactions between age and test number were not significant $[F(2,112)=0.01]$, and the main effect of addend was not significant $[F(5,280)=$ $1.67, p>.1$; see Figure 4].

\section{Response Times}

RTs by phase, addend, and strategy (computes vs. retrieves) are shown in Figure 5 (upper panel, younger adults; lower panel, older adults). Only the data for late-young and late-old subgroups are reported, because early learners produced too few compute responses for this breakdown. The plots suggest that RTs were longer for computes than for retrieves and that RTs for computes increased as a function of addend size, whereas RTs for retrieves were unaffected by addend size. These observations were confirmed in a series of linear regression analyses using group data, with age, addend, and phase as predictors. For the late-young learners, the regression model was significant for computes $[F(2,15)=$ 86.70, $\left.M S_{\mathrm{e}}=64515.6, p<.001 ; R^{2}=.91\right]$ but was not significant for retrieves $(F<1)$. For computes, addend 


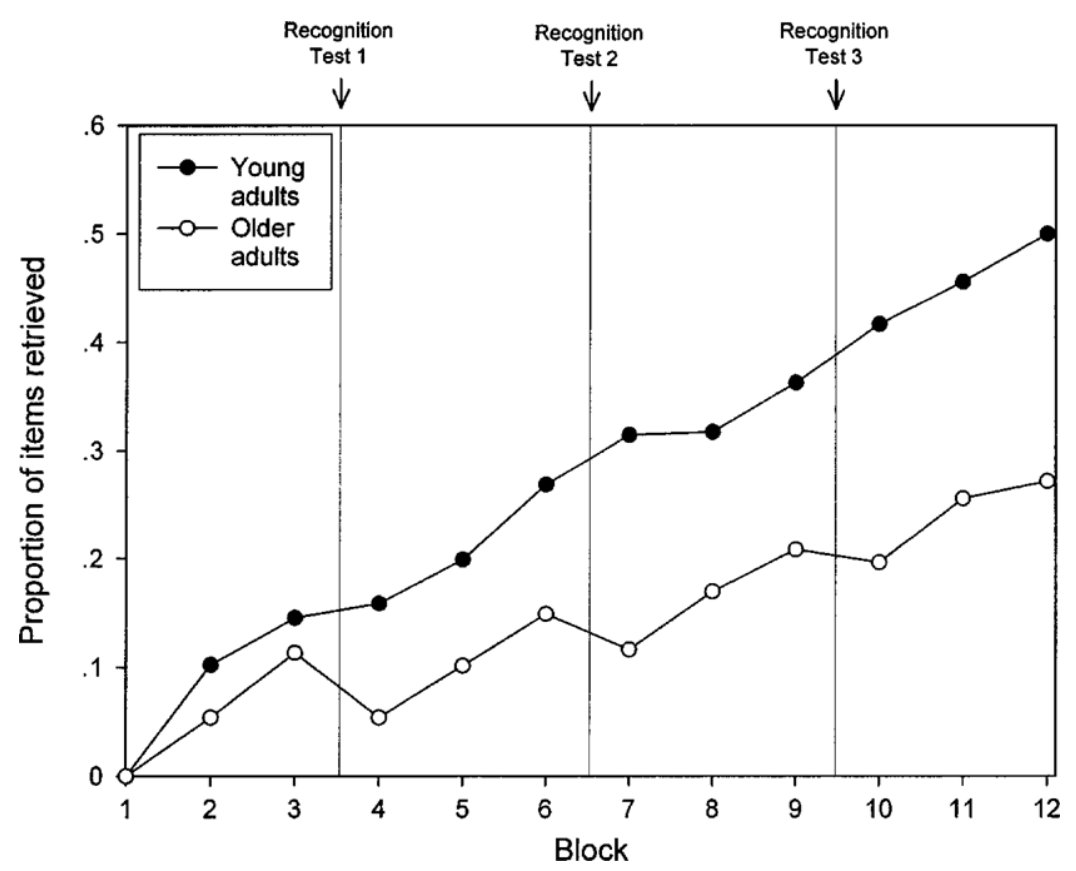

Figure 3. Proportions of items retrieved as a function of blocks for younger and older adults in Blocks 1-12. The vertical lines indicate the points of administration of the recognition tests.

was a significant predictor of RTs $(t=13.16, p<.001)$, and phase was not. Similarly, for the late-old learners, the regression model was significant for computes $\left[F(2,15)=103.37, M S_{\mathrm{e}}=105,517.8, p<.001 ; R^{2}=\right.$ $.92]$, but not for retrieves $(F<1)$. For the late-old learners, addend was a significant predictor of RTs $(t=0.96$, $p<.001$ ), and phase was not.

Age differences in these RTs were examined in two analyses that collapsed across phase. For retrieves, data were also collapsed across addend. Retrieval times were longer for older adults $(2,734 \mathrm{msec})$ than for younger adults $[1,886 \mathrm{msec} ; t(35)=3.67, p<.001]$. Computation times were regressed onto addend, separately for each participant. Because addend codes were recentered around the mean (addends $-2.5,-1.5,0.5,1.5,2.5$, and 3.5 ), the resulting intercepts corresponded to the overall mean for the participant. Slopes for younger adults (408.9) and older adults (505.8) did not differ reliably $[t(35)=1.28, p=.2]$. Unlike the slopes, intercept values for younger adults $(3,916 \mathrm{msec})$ and older adults $(4,804 \mathrm{msec})$ did differ significantly $[t(35)=2.38, p<$ $.05]$. Note that these age effects pertain only to the late-young and late-old subgroups, and not to all the participants.

\section{DISCUSSION}

In a number of studies of cognitive skill acquisition (e.g., Compton \& Logan, 1991; Delaney et al., 1998; Haider \& Frensch, 2002; Rickard, 1997), it has been shown that there is a shift in the way in which solutions to repeated problems are obtained. The shift is to retrieval of prior solutions, allowing the learner to avoid a lengthy computation. We asked in this experiment whether the ease with which problem solutions were acquired was affected by the difficulty of the computation. Problem difficulty was operationalized by the size of the addend in alphabet arithmetic problems, and solution learning was assessed by the participants' reports following each trial, as to whether they had computed or retrieved.

Three different outcomes seemed plausible in light of current theories of skill learning and memory. First, in terms of the two most developed theories of skill learning (Logan, 1988, 1992; Rickard, 1997), memory trace formation is conceptualized as automatic and effortless; in that case, problem difficulty should have had no effect on item learning. Second, in terms of depth-of-processing theory (e.g., Craik, 2002), computation may be akin to a relatively deep orienting task at encoding; in that case, lengthy computations should have enhanced item learning. Third, in terms of resource-based theories of working memory (e.g., J. R. Anderson, Reder, \& Lebiere, 1996) and cognitive aging (e.g., Salthouse, 1996), the computation process may draw on resources used for trace formation or may disrupt its consolidation; in that case, item difficulty should interfere with item learning.

The findings pointed clearly to the last of these alternatives: Harder problems were learned later. This outcome was replicated in four subgroups of participants that differed markedly in their overall rate of learning. Partitioning each participant's learning record into first-third, second-third, and third-third, the tilt in 

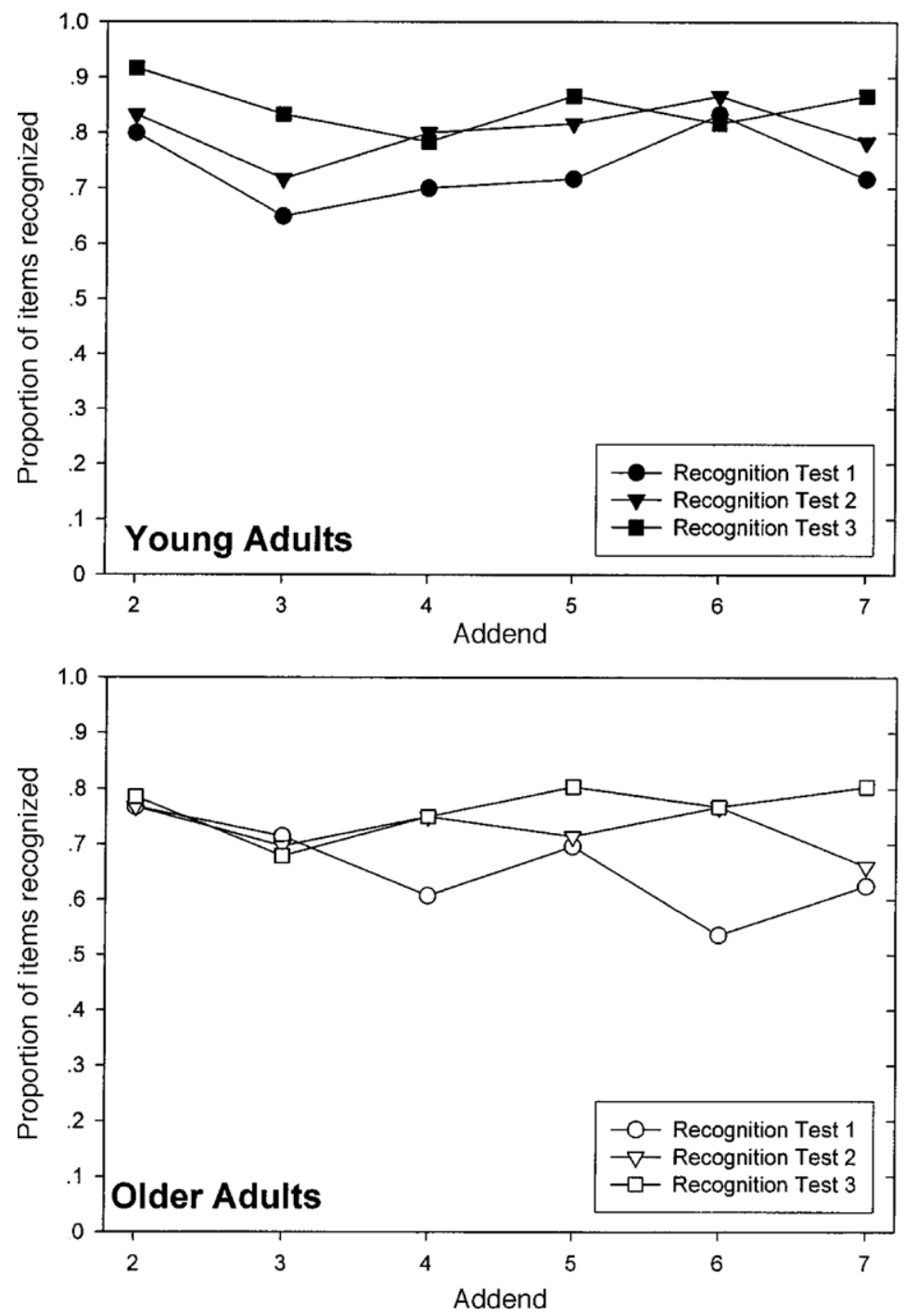

Figure 4. Item recognition as a function of test and addend for younger and older adults.

favor of easier items was the same in all the subgroups at each phase of learning.

These findings have several implications for the interpretation of skill acquisition data and theory. Datawise, one might expect to observe item-learning effects between experiments, as well as within experiments, when comparing outcomes from tasks that differ in difficulty. For comparison, we derived data from three tasks that could be cast in a common format. In these experiments, a fixed set of problems was presented for 10 repetitions, at which point performance on novel problems was also assessed. Although these experiments did not measure item learning directly through the use of strategy probes, the magnitude of item learning could nonetheless be inferred through a comparison of the RTs. Let $\Delta_{\mathrm{R}}$ be the speedup in response to repeated items given by $\mathrm{RT}_{1}-\mathrm{RT}_{10}$, the difference between the times for Block 1 and the times for Block 10. Let $\Delta_{\mathrm{N}}$ be the speedup to novel items. The latter value has a clear-cut meaning: It defines the improvement in computation with training (together with the improvement in the perceptual-motor operations common to both novel and repeated items). It is reasonable to propose the quantity $\Delta_{\mathrm{R}}-\Delta_{\mathrm{N}}$ as a proxy for the amount of item learning; it reflects that portion of the repetition effect for repeated items that remains once the portion due to algorithmic speedup is removed. For comparisons across tasks, this quantity can be normalized by $\Delta_{R}$ : Thus, $\left(\Delta_{R} \times \Delta_{N}\right) / \Delta_{R}$ represents a proportional measure of the magnitude of item learning based on RT information.

This statistic was computed for three sets of published data. The most complex of the tasks required a four-digit number to be reduced to a single digit by the sequential application of pairwise synthetic arithmetic operations 

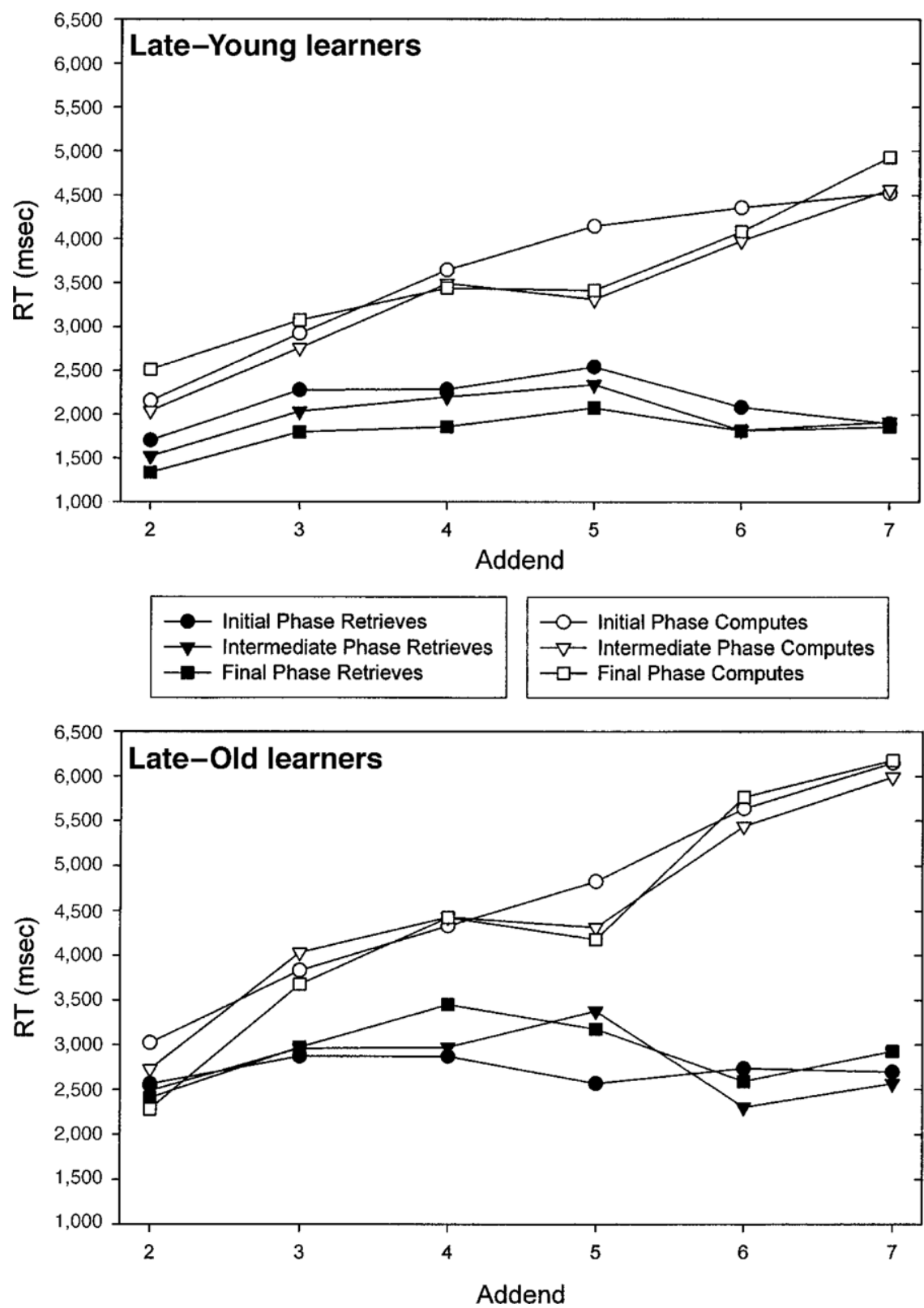

Figure 5. Response times (RTs) by phase and addend for younger adults (upper panel) and older adults (lower panel). Only late-learner data are presented, because early learners did not provide enough data points for a reliable analysis.

(Woltz, Gardner, \& Bell, 2000). In Woltz et al.'s (2000) Experiment 3, the Block 1 time for a set of problems averaged 5,250 msec. RTs dropped to less than half that value after 10 repetitions $(2,125 \mathrm{msec})$. At that point, the response to novel items was nearly as fast $(2,200 \mathrm{msec})$. Thus, about $98 \%$ of the gain in latencies with training could be attributed to a speedup in the computation, and only $2 \%$ to item-specific learning. In an earlier study, Woltz, Bell, Kyllonen, and Gardner (1996, Experiment 3) reported very nearly the same values, using 5.4 repetitions of the same stimulus set.
Compare Woltz et al.'s (1996; Woltz et al., 2000) results with those from a much easier task, in which participants were required to flag letter strings as nonwords (Logan, 1988, Experiment 1, nonword condition). The initial latency for this task was about $695 \mathrm{msec}$ and dropped to about $525 \mathrm{msec}$ after 10 repetitions of the stimulus set. At that point, $49 \%$ of the overall gain was due to algorithmic speedup, and 51\% to item learning. The task of flagging letter strings as words was even easier; RTs in Logan's word condition dropped from about 587 to $500 \mathrm{msec}$ over 10 repetitions. At that point, $14 \%$ 
of the gain was due to algorithmic speedup, and $86 \%$ was item specific.

Although the relation of these cases to our findings is somewhat weakened because we are forced to infer item learning from latencies, rather than from strategy reports, the order of these three outcomes is consistent with our findings: Lengthy computations serve to attenuate item learning during skill training. Rickard (1997, Experiment 2) performed an experiment very similar to ours that did use strategy probes. Eight alphabet arithmetic problems having addends of 3,5 , and 7 were presented 80 times. However, retrieval proportions were not tracked by addend. The reported analysis of total retrievals would have been insensitive to small differences in learning rates by addend, because the measures were all close to ceiling over Blocks 40-80.

In terms of implications for theories of cognitive skill acquisition, our results point to the need to recast item learning as an effortful process. This change in status need not challenge the fundamentals of either Logan's (1988, 1992) instance theory or Rickard's (1997) components power laws (CMPL) theory. The machinery is present in both models to take into account the effects of computational load found here.

The CMPL model of Rickard (1997) takes the form of a simulated neural network. The network is organized so that presentation of a stimulus item activates two parallel pathways, one pathway culminating in a calculated response and the other culminating in a prestored response. Mutually inhibitory links interconnect rule nodes and retrieval nodes. The node on one side of a link acts to suppress the node on the other side. When one of the two inhibitory signals reaches a threshold value, it shuts down the opposing pathway, and the network resolves to a single pathway. Only the dominant process, be it a calculation or a retrieval, is completed. The calculation pathway, if potentiated, proceeds in a series of steps, each activating preexisting knowledge required by the algorithm. In contrast, the retrieval pathway is completed in a single step.

Learning involves incremental strengthening of the links that connect input nodes to interior nodes at the conclusion of the trial. The amount of strengthening varies from item to item-an aspect of the CMPL model designed to capture chance mnemonic differences in item learnability. Tying together the item learnability parameter and the computational step count parameter in an inverse relation could accommodate the findings of the present experiment.

In Logan's $(1988,1992)$ instance theory, each presentation of an item is recorded in a distinct memory trace. Subsequent presentations activate every prior trace, and the first trace to reach a fixed threshold value terminates the retrieval process. Stimulus presentation also activates a computation process concurrent with the retrieval process. Both computation times and retrieval times vary stochastically, and the first process to terminate deactivates the competing process and concludes the trial. Skill is embodied in the accumulation of memory traces. As traces grow more numerous, the likelihood increases that one of them will become fully activated before rule completion.

With respect to our results, note first of all that the intrinsic dynamics of the computation-retrieval "race" predict an outcome opposite to the one we found: Proliferating memory traces will overtake a long computation earlier in training than a short computation (see Logan, 1988, Figure 3, simulation results). This outcome, then, needs to be reversed. This could be done by applying a memory efficiency parameter that serves to fix the proportion of trials on which an instance is stored, as was suggested but not used by Logan $(1988,1992)$. Linking the mean computation time to memory efficiency in an inverse relation can accommodate our findings. Although the patches discussed above for both Rickard's (1997) and Logan's $(1988,1992)$ theories are somewhat ad hoc, the inverse relationships we propose are very much in the spirit of capacity limits, as formalized in a principled way in the context of the development of ACT-R by J. R. Anderson et al. (1996).

Participants' knowledge of items, as distinct from their solutions, was assessed by three interpolated recognition tests. Item information differed dramatically from solution information in two ways. First, it was acquired very rapidly. On the first recognition test following three blocks of training, the mean recognition score for the younger participants was 8.8 of 12 items, whereas the mean for reported retrievals was 1.74 of 12 solutions in Block 3. For the older participants, the mean recognition score was 7.9, and the mean for reported retrievals was 1.36 in Block 3. Second, item information was not modulated by addend. Load-free acquisition is characteristic of familiarity-based judgments (e.g., Mangels, Picton, \& Craik, 2001).

Our results also bear on the description of the effects of aging on skill learning. For the most part, the performance of the older adults paralleled that for the younger adults, superimposed on characteristic age reductions in both the memory-based measures and the speed measures. Computational load did not interact with age, as indexed by the slope of the enumeration function (Figure 5). This particular result coincides with other aging studies that have suggested that lexically or semantically based operations, such as word priming or arithmetic, show little if any loss with age (see, e.g., Salthouse, 1996; Verhaeghen \& Salthouse, 1997). The slowing ratio of 1.2 per addend in computation is typical of age deficits in lexical processes, such as letter counting (Myerson, Hale, Chen, \& Lawrence, 1997). Although this finding is contrary to the results of previous studies reporting age deficits in skill learning (e.g., Touron et al., 2001) and to the prediction that the item difficulty effect would interact with age, so that older adults would find hard items disproportionately more difficult to learn, it is possible that the computations were not difficult enough or sensitive enough to show the relatively limited processing resources of older adults. Note that at least some, and possibly all, of the large reduction in the overall rate at 
which item-solution associations were acquired and retrieved by the older adults can be attributed to the disruptive effect of the interpolated tests for the older adults. Further work in which the mechanisms of learning, memory, and attention that underlie aging effects in skill acquisition are examined is needed.

\section{REFERENCES}

Anderson, J. R., Reder, L. M., \& Lebiere, C. (1996). Working memory: Activation limitations on retrieval. Cognitive Psychology, 30, 221-256.

Anderson, N. D., Craik, F. I. M., \& Naveh-Benjamin, M. (1998). The attentional demands of encoding and retrieval in younger and older adults. Psychology \& Aging, 13, 405-423.

BARRouIllet, P., \& FAYOL, M. (1998). From algorithmic computing to direct retrieval: Evidence from number and alphabetic arithmetic in children and adults. Memory \& Cognition, 26, 355-368.

Castel, A. D., Benjamin, A. S., Craik, F. I. M., \& Watkins, M. J. (2002). The effects of aging on selectivity and control in short-term recall. Memory \& Cognition, 30, 1078-1085.

Cerella, J. (1990). Aging and information-processing rate. In J. E. Birren \& K. W. Schaie (Eds.), Handbook of the psychology of aging (3rd ed., pp. 201-221). New York: Academic Press.

Charness, N., \& Campbell, J. I. D. (1988). Acquiring skill at mental calculation in adulthood: A task decomposition. Journal of Experimental Psychology: General, 117, 115-129.

Cherry, K. E., \& PArk, D. C. (1993). Individual differences and contextual variables influence spatial memory in younger and older adults. Psychology \& Aging, 8, 517-526.

Compton, B. J., \& Logan, G. D. (1991). The transition from algorithm to retrieval in memory-based theories of automaticity. Memory \& Cognition, 19, 151-158.

Craik, F. I. M. (2002). Levels of processing: Past, present, and future. Memory, 10, 305-318.

Craik, F. I. M., Naveh-Benjamin, M., Ishaik, G., \& Anderson, N. D. (2000). Divided attention during encoding and retrieval: Differential control effects? Journal of Experimental Psychology: Learning, Memory, \& Cognition, 26, 1744-1749.

Delaney, P. F., Reder, L. M., Staszewsi, J. J., \& Ritter, F. E. (1998). The strategy-specific nature of improvement: The power law applies by strategy within task. Psychological Science, 9, 1-7.

Ekstrom, R. B., French, J. W., Harmon, H. H., \& Dermen, D. (1976). Manual and kit of factor-referenced cognitive tests. Princeton, NJ: Educational Testing Service.

Haider, H., \& Frensch, P. A. (2002). Why aggregated learning follows the power law of practice when individual learning does not. Journal of Experimental Psychology: Learning, Memory, \& Cognition, 28, 392406.

Hashtroudi, S., Chrosniak, L. D., \& Schwartz, B. L. (1991). Effects of aging on priming and skill learning. Psychology \& Aging, 6 , 605-615.

JenKIns, L., \& Hoyer, W. J. (2000). Instance-based automaticity and aging: Acquisition, reacquisition, and long-term retention. Psychology \& Aging, 15, 551-565.

Kramer, A. F., Larish, J. F., \& Strayer, D. L. (1995). Training for attentional control in dual task settings: A comparison of young and old adults. Journal of Experimental Psychology: Applied, 1, 50-76.

LoGAN, G. D. (1988). Toward an instance theory of automatization. Psychological Review, 95, 492-528.

LogAN, G. D. (1992). Shapes of reaction-time distributions and shapes of learning curves: A test of instance theory of automatization. Journal of Experimental Psychology: Learning, Memory, \& Cognition, 18, 883-914.

Logan, G. D., \& KLAPP, S. T. (1991). Automatizing alphabet arithmetic: I. Is extended practice necessary to produce automaticity? Journal of Experimental Psychology: Learning, Memory, \& Cognition, 17, 179-195.

Mangels, J. A., Picton, T. W., \& Craik, F. I. M. (2001). Attention and successful episodic encoding: An event-related potential study. $\operatorname{Cog}$ nitive Brain Research, 11, 77-95.

Myerson, J., Hale, S., Chen, J., \& Lawrence, B. (1997). General lexical slowing and the semantic priming effect. Acta Psychologica, 96, 83-101.

Naveh-Benjamin, M. (2000). Adult age differences in memory performance: Tests of an associative deficit hypothesis. Journal of Experimental Psychology: Learning, Memory, \& Cognition, 26, 1170-1187.

Newell, A., \& Rosenbloom, P. S. (1981). Mechanisms of skill acquisition and the law of practice. In J. R. Anderson (Ed.), Cognitive skills and their acquisition (pp. 1-55). Hillsdale, NJ: Erlbaum.

Nosofsky, R. M., \& PAlmeri, T. J. (1997). An exemplar-based random walk model of speeded classification. Psychological Review, 104, 266-300.

ONYPER, S., \& HoYer, W. J. (2003). The number ordering test: Manual. Syracuse University, Department of Psychology.

PAlmeri, T. J. (1997). Exemplar similarity and the development of automaticity. Journal of Experimental Psychology: Learning, Memory, \& Cognition, 23, 324-354.

RICKARD, T. C. (1997). Bending the power law: A CMPL theory of strategy shifts and the automatization of cognitive skills. Journal of Experimental Psychology: General, 126, 288-310.

Rogers, W. A., Hertzog, C., \& Fisk, A. D. (2000). An individual differences analysis of ability and strategy influences: Age-related differences in associative learning. Journal of Experimental Psychology: Learning, Memory, \& Cognition, 26, 359-394.

SAlthouse, T. A. (1994). Aging associations: Influence of speed on adult age differences in associative learning. Journal of Experimental Psychology: Learning, Memory, \& Cognition, 20, 1486-1503.

SAlthouse, T. A. (1996). The processing-speed theory of adult age differences in cognition. Psychological Review, 103, 403-428.

Salthouse, T. A., \& Somberg, B. L. (1982). Skilled performance: The effects of adult age and experience on elementary processes. Journal of Experimental Psychology: General, 111, 176-207.

SiEgLER, R. S., \& LEMAIRE, P. (1997). Older and younger adults' strategy choices in multiplication: Testing predictions of ASCM using the choice/no-choice method. Journal of Experimental Psychology: General, 126, 71-92.

Sмiтн, A. (1982). Symbol digit modalities test: Manual. Revised 1982. Los Angeles: Western Psychological Services.

Strayer, D. L., \& Kramer, A. F. (1994). Aging and skill acquisition: Learning-performance distinctions. Psychology \& Aging, 9, 589-605.

Thevenot, C., Barrouillet, P., \& Fayol, M. (2001). Algorithmic solution of arithmetic problems and operands-answer associations in long-term memory. Quarterly Journal of Experimental Psychology, 54A, 599-611.

Touron, D. R, Hoyer, W. J., \& Cerella, J. (2001). Cognitive skill acquisition and transfer in younger and older adults. Psychology \& Aging, 16, 555-563.

Touron, D. R., Hoyer, W. J., \& Cerella, J. (2003). Aging of item learning in cognitive skill acquisition. Unpublished manuscript, Syracuse University.

Turner, M. L., \& ENGLE, R. W. (1989). Is working memory capacity task dependent. Journal of Memory \& Language, 28, 127-154.

Verhaeghen, P., \& Salthouse, T. A. (1997). Meta-analyses of agecognition relations in adulthood: Estimates of linear and non-linear age effects and structural models. Psychological Bulletin, 122, 231-249.

WeCHSLER, D. (1981). Wechsler Adult Intelligence Scale-Revised. New York: Psychological Corporation.

Woltz, D. J., Bell, B. G., Kyllonen, P. C., \& Gardner, M. K. (1996). Memory for order of operations in the acquisition and transfer of sequential cognitive skills. Journal of Experimental Psychology: Learning, Memory, \& Cognition, 22, 438-457.

Woltz, D. J., Gardner, M. K., \& Bell, B. G. (2000). Negative transfer errors in sequential cognitive skills: Strong-but-wrong sequence application. Journal of Experimental Psychology: Learning, Memory, \& Cognition, 26, 601-625.

(Manuscript received February 9, 2003; revision accepted for publication August 5, 2003.) 